Einführung in das Schwerpunktthema

\title{
Öko-Effizienz - mehr Fragen als Antworten?
}

D Von Gerd U. Scholl und Jens Clausen as Thema Öko-Effizienz ist in aller Munde. Mit diesem schillernden Begriff verbinden umwelt- wie auch wirtschaftspolitische Akteure die Hoffnung, „zwei Fliegen mit einer Klappe zu schlagen": Die Realisierung eines angemessenen ökonomischen Wohlstandsniveaus bei gleichzeitiger Erhaltung der natïrlichen Lebensgrundlagen. In der Definition des World Business Council for Sustainable Development heißt das: „Eco-efficiency is reached by the delivery of competitively priced goods and services that satisfy human needs and bring quality of life, while progressively reducing ecological impacts and resource intensity throughout the life cycle, to a level at least in line with the earth's carrying capacity" (1). Ein Indikator für die Attraktivität des Themas sind die vielen internationalen Tagungen, die in jüngster Zeit dazu stattgefunden haben, und eine fast nicht mehr überschaubare Menge an Veröffentlichungen (vgl. Literatur zum Schwerpunkt, S. 14). Doch je näher man diesen Ansatz betrachtet, auf desto mehr Fragen stößt man (2). Dies gilt sowohl für die gesamtwirtschaftliche als auch für die einzelwirtschaftliche Dimension (für eine Einordnung aus der Unternehmensperspektive vgl. den Beitrag von Schaltegger). Beide Sichtweisen werden von den weiteren Schwerpunktbeiträgen thematisiert.

\section{- Was ist der "added value"?}

Eine der Grundannahmen bei der Diskussion um Öko-Effizienz ist die, daß die Wirtschaft nur dann einen ressourcenschonenden Entwicklungspfad einschlagen kann, wenn der Umstieg mit einem ökonomischen Zusatznutzen verbunden ist. Dieser wird in der Regel als ,added value" bezeichnet, ein Begriff, der zwar griffig und ansprechend scheint, dem es aber nichtsdestotrotz bisher an Klarheit und häufig auch Operationalisierbarkeit fehlt. Der ,added value“ für die Anbieter öko-effizienter Produkte und Dienstleistungen liegt beispielsweise in höheren Gewinnmargen, verbessertem Unternehmensimage, intensiveren Kundenbindungen oder auch in größeren Potentialen, sich an Änderungen des umweltgesetzlichen Rahmens anzupassen. Aus Sicht der Nachfrager trägt der ,,added value" dazu bei, das Ausmaß der Bedürfnisbefriedigung zu steigern. Doch insbesondere hier bieten die Wirtschaftswissenschaften keine eindeutige Methodologie zur Messung an (3). Dies hat ferner zur Konsequenz, daß Operationalisierungsansätze, wie beispielsweise das vom Wuppertal-Institut entwickelte MIPS-Konzept (Materialintensität pro Serviceeinheit), auf Hilfskonstrukte zurückgreifen, die den Nutzen auf physikalisch meßbare Leistungseinheiten reduzieren und damit die zugrundeliegenden Bedürfnisse und individuellen Präferenzen tendenziell ausblenden. Auf diesen letzten Aspekt geht Diekmann in seinem Beitrag ein, indem er die postulierte Trennschärfe von Effizienzansätzen (technisch induzierte Umweltverbesserungen) und Suffizienzansätzen (verhaltensinduzierte Umweltverbesserungen) kritisiert.

\section{- Wie ökologisch ist Öko-Effizienz?}

Die Umweltvorteile öko-effizienter Wertschöpfungsprozesse resultieren aus einer Verbesserung des Verhältnisses von Inputs zu Outputs. Weniger Ressourcen werden benötigt, um eine Produkt- oder Dienstleistungseinheit zur Verfügung zu stellen. Doch zum einen stößt man hier bereits auf systemimmanente Optimierungsgrenzen, die sich aus den vorhandenen Technologien ergeben (selbst ein Dreiliterauto ist auf materielle Infrastrukturen wie Straßen und Parkhäuser angewiesen). Hierauf geht Reijnders in seinem Beitrag näher ein.

Zum anderen können Problemverlagerungen entlang des Lebenszyklusses (z.B. Leichtbauweise aus Aluminium, die zur Senkung des Ressourcenverbrauchs während der Nutzung führt, jedoch einen höheren Energieinput zur Herstellung des Werkstoffes benötigt) oder auch Mengeneffekte, die die Umweltgewinne pro Produkteinheit durch eine mengenmäßige Steigerung des Produktdurchsatzes überkompensieren (vgl. den Beitrag von Stablmann/Clausen), die ökologische Bilanz beein- trächtigen (4). Stabl/Fleig weisen deshalb in ihrem Artikel daraufhin, daß „nur unter bestimmten Rahmenbedingungen die Konzepte ökologische Entlastungen mit sich bringen", von einem ökologischen Automatismus daher also kaum die Rede sein kann (vgl. dazu auch den Beitrag von de Bruyn). Einen durchaus diskussionswürdigen Ansatz stellt in diesem Zusammenhang das Mobilitätskonzept des Stadtautos Smart dar, das Hoßfeld in seinem Beitrag darstellt. Erst ein systemischer Ansatz, der unterschiedliche, möglicherweise öko-effiziente Verkehrsträger in einer bedarfsorientierten Art und Weise mit einander verknüpft, kann die hochgesteckten Ziele einer umweltverträglicheren Mobilität erreichen.

\section{Arbeitsplätze durch Öko-Effizienz?}

Zahlreiche Untersuchungen zum Thema Umweltschutz und Arbeit liegen vor. Zur Zeit geht man von fast einer Million umweltschutzinduzierter Arbeitsplätze in Deutschland aus (5). Doch hierbei handelt es sich meistens um Beschäftigung, die auf additive Umweltschutzmaßnahmen zurückgeht (z.B. Herstellung von End-of-pipe Technologien, Altlastensanierung, Recycling von Altgeräten). In dem Maße wie Umweltschutz jedoch dem Vorsorgeanspruch gerecht wird, und dies gilt sicherlich auch für das Öko-Effizienz-Paradigma, verlagert sich der Beschäftigungsimpuls auf integrierte Maßnahmen, deren Effekt in dieser Hinsicht weniger eindeutig ist (6).

Die Untersuchung der Beschäftigungswirkungen beispielsweise von Strategien der Nutzungsdauerverlängerung und Nutzungsintensivierung von Produkten, die im Rahmen der Öko-EffizienzDebatte eine prominente Stellung einnehmen (Stichwort Serviceökonomie), unterstreicht die Einschätzung, daß nur unter sehr optimistischen Annahmen davon auszugehen ist, daß die negativen Beschäftigungseffekte auf Ebene der Produktfertigung inklusive aller Vorlieferstufen, die die Folge eines insgesamt reduzierten Güterbedarfs sind, durch neu entstehende Beschäftigungspotentiale in der Nutzungs- und Nachnutzungsphase überkompensiert werden können (7). Vor dem Hintergrund zunehmender Marktsättigungstendenzen und steigender Preissensibilität bleibt zudem für die Anbieter ökoeffizienter Dienstleistungskonzepte nur ein geringer Spielraum: „Für die Arbeitsplätze bedeutet dies, entgegen den teilweise hohen Erwartungen, daß konkurrenzfähige ökologi- 
sche Dienstleistungen zukünftig weniger arbeitsplatzintensiv sein werden und zudem verlagerbar sind“" (8).

\section{Was ist die politische Dimension?}

Das Thema Öko-Effizienz hat auch eine aktuelle und hochpolitische Seite. Der Blick zurück auf die Entwicklung der Umweltbewegung seit den 70er Jahren läßt erkennen, daß sich ökologische Ideale häufig, wenn nicht immer, mit einer Infragestellung kultureller Werte, Lebensstile, Wirtschaftsweisen und auch der Eigentumsverhältnisse gekoppelt haben. Die Tiefenökologie, die Debatte um nachhaltige Regionalentwicklung und auch Gedanken wie sie z.B. von E.F. Schumacher geäußert wurden (9), dies alles sind keine rein ökologischen Konzepte, sondern Konzepte tiefgreifender gesellschaftlicher Veränderungen. Es wundert kaum, daß die Umsetzung solcher Gedanken nicht im Interesse maßgeblicher wirtschaftlicher Akteure war. Da die Strategie, Vorstellungen zum konsequent ökologischen und damit gesellschaftlichen Strukturwandel entweder zu ignorieren oder gar mehr oder weniger offen zu bekämpfen, sich spätestens in den 80er Jahren als untauglich herausstellte, war eine neue Strategie gefragt. Richard Welford benennt den Öko-Modernismus mit seinem Flaggschiff, der Öko-Effizienz, als diese neue Strategie und unterstellt ihr die Absicht, damit die Umweltbewegung „kapern“ zu wollen (10).

Diese Strategie setzt der Uneinigkeit unterschiedlicher Flügel der Umweltbewegung eine vermeintlich wirksame und klare Orientierung entgegen. Gleichzeitig verschiebt sich der Schwerpunkt notwendiger Änderungen in der Gesellschaft von kulturell determinierten Lebensstilen und festgefuigten Wirtschaftsstrukturen auf eine durch Kapital und Industrie kontrollierbare, ökonomisch-ökologische Effizienzstrategie (Öko-Effizienz als ,add-on feature of business-as-usual"). Vom Gegner der Umweltbewegung wandelt die Industrie sich so zum zentralen Akteur der ökologischen Erneuerung. Dies geschieht aber um den Preis einer erstaunlichen Verengung des Zielfeldes.

Ausgeblendet bleiben die spirituellen Dimensionen der Ökologiebewegung, z.B. die Gedanken zum Eigenwert der Natur. Aber auch Subsistenz, Konsumverzicht und systemische Lösungen ökologischer Probleme, wie sie sich z.B. im Mobilitätsbereich durch die Abkehr vom Auto als Eigentum andeuten, liegen nicht im Spektrum des von der Industrie Gewollten. Jegliches Sägen an den Säulen des Freihandels, des technischen Fortschrittes, des Wirtschaftswachstums und der kontinuierlichen Verbesserung - in kleinen Schritten und ohne die Systemfrage zu stellen gilt es der Industrie zu vermeiden.

Welford beurteilt die Rolle des industriell geprägten Öko-Effizienz-Konzeptes als Beitrag zur nachhaltigen Entwicklung ausgesprochen kritisch in fünf Punkten (Übersetzung durch die Verfasser):

- Mit Öko-Effizienz lassen sich einige Umweltbelastungen reduzieren, aber das Konzept kommt nicht an die wirklichen Ursachen der Umweltkrise heran. Eine Infragestellung der Konsumgewohnheiten, der materiellen Orientierung, des Wachstums und sich wandelnder Lebensstile sind alle im Rahmen der Ökologiedebatte notwendig, erfolgen aber nur in der Umweltbewegung, nicht in der industriekontrollierten Öko-Effizienz-Debatte.

- Öko-Effizienz und Öko-Modernismus wurden beide aus der Industrie heraus entwickelt. Sie sind nicht mit einer nachhaltigen Entwicklung in Übereinstimmung zu bringen, weil sie untrennbar mit Wachstum Globalisierung und Freihandel (als Gegenpol zu fairem Handel) verbunden sind.

- Die Arroganz, die oft mit dem Öko-Effizienz Konzept verbunden ist, könnte ein Hindernis des Fortschritts auf der ökologischen Agenda sein. Öko-Effizienz wird hier zu oft selbst als Ziel gesehen. Die Ironie dabei ist, daß uns Fortschritte der Öko-Effizienz zwar ein Stück des Weges zur nachhaltigen Entwicklung voranbringen können, daß die damit verbundene Zementierung der falschen Grundlagen des Wirtschaftssystems danach aber weiteren Fortschritt verhindern könnte

- Öko-Effizienz beschäftigt sich nicht mit den sozialen und ethischen Fragen, die von der Umweltbewegung aufgeworfen wurden. ÖkoEffizienz kann schon deswegen immer nur zu einer Teillösung führen.

- Die wirtschaftliche Macht und Arroganz, die hinter dem Öko-Effizienz-Konzept stehen, verhindern heute aktiv tiefergehende Debatten über alternative Wege zur nachhaltigen Entwicklung. Diesen Verlust sollten wir alle bedauern.

Im Rahmen des Diskurses der ökologischen Ökonomie sollten also einzelne Konzepte, besonders wenn sie derart absichtsvoll von strukturell nicht an tiefergehender Veränderung interessierten Akteuren unterstützt werden, immer wieder hinterfragt und auf ihre wirkliche Wirksamkeit hin untersucht werden. Dazu mag dieses Heft ein Beitrag sein.

\section{Anmerkungen}

(1) WBCSD: Achieving Eco-Efficiency in Business. Report prepared for the Second Antwerp Efficiency Workshop, March 14-15, 1995.

(2) Eine Vielzahl unterschiedlicher Aspekte, die mit Blick ouf öko-effiziente Dienstleistungskonzepte im Konsumgütersektor relevant sind (z.B. Vebraucherakzeptanz, ökologische Entlastungspotentiale), werden im Rahmen eines laufenden IÖW-Projektes untersucht: „Neue Nutzungskonzepte für Produkte", Informationen unter

http://www.ioew.de/nutzungskonzepte.

(3) Vgl. Heiskanen, Eva/Mari Niva: A Bird's Eye View on Users and Usefulness. In National Consumer Research Center (Ed.): Take a Look at the User - Perspectives on Users and Usefulness, Helsinki 1996.

(4) Vgl. hierzu auch Hirschl, Bernd/Gerd Scholl/Frank Tibjtanzl: Öko-Effizienz auf dem Prüfstand. Grundlagen der Bewertung neuer Nutzungskonzepte für Produkte. In: Ökologisches Wirtschaften 5/1998, S. 30-31.

(5) Vgl. Umweltbundesamt (Hrsg.): Umweltschutz und Beschäftigung. Brückenschlag für eine lebenswerte Zukunft. Berlin 1997.

(6) Vgl. Pfeiffer, Friedhelm/ Klous Rennings: Weder Jobkiller noch Jobwunder. Die Beschäftigungswirkungen des integrierten Umweltschutzes. In: Ökologisches Wirtschaften, 2/1999, S. 26-27.

(7) Vgl. Scholl, Gerd: Beschäftigungsimplikationen einer Verlängerung und Intensivierung der Produktnutzung. Unveröffentlichtes Manuskript erstellt im Rahmen des Verbundprojekts "Arbeit \& Ökologie" am Wissenschaftszentrum Berlin, Stuttgart/Heidelberg 1999.

(8) Behrendt, Siegfried/ Ralf Pfitzner: Ökologische Dienstleistungskonzepte als Elemente einer Kreislaufwirtschaft, Berlin 1998.

(9) Vgl. Schumacher, Ernst Friedrich: Small is Beautiful. Die Rückkehr zum menschlichen Maß. (z.B. Deukalion 1995)

(10) Vgl. Welford, Richard: Hijacking Environmentalism? Corporate Responses to Sustainable Development, Vortrag auf der Togung Industry and Environment, Aarhus 1996, sowie sein gleichnamiges, im Earthscan Verlag, London erschienenes Buch.

\section{Die Autoren}

Gerd U. Scholl ist Leiter des Forschungsfeldes "Ökologische Produktpolitik", Jens Clausen arbeitet im Forschungsfeld "Ökologische Unternehmenspolitiik" des IÖW. Kontakt: Gerd U. Scholl, IÖW-Regionalbüro Baden-Württemberg, Bergstr. 7, 69120 Heidelberg. Tel. 06221/ 64916-3, Fax 06221/ 27060, E-mail: Gerd.Scholl@heidelberg.ioew.de 
(c) 20I0 Authors; licensee IÖW and oekom verlag. This is an article distributed under the terms of the Creative Commons Attribution Non-Commercial No Derivates License (http://creativecommons.org/licenses/by-nc-nd/3.o/), which permits unrestricted use, distribution, and reproduction in any medium, provided the original work is properly cited. 